\title{
Injectable Hydrogel Based on Modified Gelatin and Sodium Alginate for Soft-Tissue Adhesive
}

\author{
Yuhang Xing ${ }^{1 \dagger}$, Xueqin Qing ${ }^{2 \dagger}$, Hao Xia ${ }^{1 \dagger}$, Shiqi Hao ${ }^{1}$, Haofang Zhu ${ }^{1}$, Yiyan $\mathrm{He}^{1,3,4}$, \\ Hongli Mao ${ }^{1,3,4 *}$ and Zhongwei Gu ${ }^{1,3,4 *}$
}

${ }^{1}$ Research Institute for Biomaterials, Tech Institute for Advanced Materials, College of Materials Science and Engineering, Nanjing Tech University, Nanjing, China, ${ }^{2}$ Department of Pediatrics, Shanghai General Hospital, School of Medicine, Shanghai Jiao Tong University, Shanghai, China, ${ }^{3}$ NJTech-BARTY Joint Research Center for Innovative Medical Technology, Nanjing, China, ${ }^{4}$ Suqian Advanced Materials Industry Technology Innovation Center of Nanjing Tech University, Nanjing, China

OPEN ACCESS

Edited by:

Houjuan Zhu,

Institute of Materials Research and

Engineering ( $A * S T A R)$, Singapore

Reviewed by:

Shasha He,

Nanyang Technological University,

Singapore

Xiaojun Zhou,

Donghua University, China

*Correspondence:

Hongli Mao

h.mao@njtech.edu.cn

Zhongwei Gu

zwgu1006@hotmail.com

tThese authors have contributed equally to this work and share first authorship

\section{Specialty section: \\ This article was submitted to \\ Nanoscience, \\ a section of the journal \\ Frontiers in Chemistry}

Received: 19 July 2021

Accepted: 02 August 2021

Published: 22 September 2021

Citation:

Xing Y, Qing X, Xia H, Hao S, Zhu H,

He Y, Mao H and Gu Z (2021)

Injectable Hydrogel Based on Modified

Gelatin and Sodium Alginate for Soft-

Tissue Adhesive.

Front. Chem. 9:744099.

doi: 10.3389/fchem.2021.744099
To assist or replace the traditional suture techniques for wound closure, soft-tissue adhesives with excellent adhesion strength and favorable biocompatibility are of great significance in biomedical applications. In this study, an injectable hydrogel tissue adhesive containing adipic acid dihydrazide-modified gelatin (Gel-ADH) and oxidized sodium alginate (OSA) was developed. It was found that this tissue adhesive possessed a uniform structure, appropriate swelling ratio, good injectability, and excellent hemocompatibility and cytocompatibility. The adhesion capacity of the developed adhesive with optimized component and concentration was stronger than that of the commercial adhesive Porcine Fibrin Sealant Kit. All these results suggested that the developed hydrogel was a promising candidate for a soft-tissue adhesive.

Keywords: soft-tissue adhesive, sodium alginate, gelatin, Schiff base reaction, hydrogel

\section{INTRODUCTION}

Rapid hemostasis and effective wound closure are very important in wound repair. Conventional suturing is usually time consuming and cannot close the wound immediately, which can lead to a variety of pathologic scenarios, such as tissue morbidities and mortalities (Geng et al., 2020; Zhu et al., 2020). Sutureless wound closure, which primarily utilizes medical adhesives, shows adequate ability in wound sealing and healing with less pain and scars (Gowda et al., 2020; Zhou et al., 2021). In addition, compared to conventional suture, it is more acceptable by the patient. Therefore, there are different types of medical adhesives have been developed in recent years. However, most of the available adhesives have certain limitations. For example, fibrin glue has favorable biocompatibility and can facilitate wound repair, but it cannot be used alone because of its unreliable mechanical and adhesion capacity. Besides, it is rather expensive and may lead to a risk of blood-borne disease or virus infections (Du et al., 2020; Gao et al., 2020). In comparison with fibrin glue, cyanoacrylates have much stronger adhesion capacity, but the excessive heat and the cytotoxic byproducts produced in the fast polymerization process during bonding are a major concern (Lv et al., 2021). Additionally, their poor elasticity cannot accommodate the movement of soft tissues under a highly dynamic physiological environment (Li et al., 2020; Zhao et al., 2021). Therefore, development of ideal medical adhesives with good biocompatibility, sufficient mechanical property, tunable biodegradability, and rapid and strong adhesion has a great application value in wound closure and healing (Neuffer et al., 2004; Gillman et al., 2020). 
Herein, by mimicking the composition of the extracellular matrix (ECM) of native tissues, a strategy to prepare adhesives with good biocompatibility and multiple functions, from polypeptide and polysaccharide, was proposed (Yang et al., 2017; Ke et al., 2020). Firstly, gelatin was modified with adipic acid dihydrazide (Gel-ADH) and sodium alginate was oxidized (OSA) with $\mathrm{NaIO}_{4}$. Then, the hydrogel adhesive was prepared through the Schiff base reaction between Gel-ADH and OSA. The results showed that the Gel-ADH/OSA hydrogel had an appropriate swelling ratio, good injectability, excellent biocompatibility, and improved adhesion capacity.

\section{MATERIALS AND METHODS}

\section{Materials}

Sodium alginate (low viscosity), gelatin (from porcine skin), and sodium periodate $\left(\mathrm{NaIO}_{4}\right)$ were purchased from Aladdin Industrial Corporation (Shanghai, China). Dulbecco's modified Eagle medium (DMEM) and fetal bovine serum (FBS) were purchased from Life Technologies Corporation (California, United States). A Porcine Fibrin Sealant Kit was purchased from Guangzhou Bioseal Biotech Co., Ltd. All other chemicals were purchased from Sigma-Aldrich and used as received unless specified otherwise.

\section{Preparation of OSA}

Oxidized sodium alginate was synthesized as reported previously (Yuan et al., 2018). Briefly, $3.00 \mathrm{~g}$ raw sodium alginate (SA) was dissolved in ultrapure water $(300 \mathrm{ml})$ and $4.86 \mathrm{~g} \mathrm{NaIO}_{4}$ was added. After that, the mixed solution was stirred for another $4 \mathrm{~h}$ without light, and then $5 \mathrm{ml}$ ethylene glycol was added in the mixed solution to terminate the oxidation. The final product was dialyzed $(\mathrm{MWCO}=3,500 \mathrm{Da})$ for 3 days in distilled water. Finally, the product was lyophilized. To confirm the characteristic peak of the aldehyde group in OSA, a Fourier transform infrared (FTIR) spectroscope (Nicolet IS 10, Thermo Scientific, United States) was used and the measurement was performed at room temperature and recorded in the $4,000-400 \mathrm{~cm}^{-1}$ range.

\section{Preparation of Gel-ADH}

To synthesize ADH-modified gelatin, $3.00 \mathrm{~g}$ gelatin and $2.40 \mathrm{~g}$ adipic acid dihydrazide were dissolved in $300 \mathrm{ml}$ ultrapure deionized water with stirring at $55^{\circ} \mathrm{C}$. Then, $0.50 \mathrm{~g}$ EDC and $0.50 \mathrm{~g} \mathrm{HOBt}$ were added to the solution. Finally, $0.1 \mathrm{M} \mathrm{HCl}$ solution was used to adjust the $\mathrm{pH}$ of the abovementioned solution to 5 . The mixed solution was stirred overnight. The final product was dialyzed $(\mathrm{MWCO}=7,000 \mathrm{Da})$ for 5 days in distilled water (Hozumi et al., 2018). The ${ }^{1} \mathrm{H}$ NMR spectra of the Gel-ADH was measured by using a $600 \mathrm{~Hz}$ NMR spectrometer (JNM-ECZR-600 Hz, JEOL, United States )

\section{Degree of Oxidation Study}

The degree of oxidation was obtained by the titration method (Reakasame and Boccaccini, 2018). In brief, Wg OSA, $5 \mathrm{ml}$ anhydrous ethanol, and $5 \mathrm{ml}$ hydroxylamine hydrochloride solution were added in a flask. Then, the solution was heated to reflux for $3 \mathrm{~h}$ and left to set for at least another $2 \mathrm{~h}$. When the solution was cooled to room temperature, $0.1 \mathrm{M} \mathrm{NaOH}$ was used to titrate the mixture. When the color of the mixed solution became yellow, the titration was stopped and the volume $\left(\mathrm{V}_{1}\right)$ of the titrimetric $\mathrm{NaOH}$ solution was recorded immediately. Then, $5 \mathrm{ml}$ anhydrous ethanol and $5 \mathrm{ml}$ hydroxylamine hydrochloride solution were added to another flask. The previous steps were repeated and the volume $\left(\mathrm{V}_{2}\right)$ of titrimetric $\mathrm{NaOH}$ solution was recorded. The oxidation degree (OD) was calculated by the following Equation:

$$
O D(\%)=198 \times 0.1 \times \frac{V_{1}-V_{2}}{2 W} \times 100 .
$$

\section{Preparation of Gel-ADH/OSA Hydrogels}

Lyophilized OSA and Gel-ADH were dissolved in the PBS solution $(\mathrm{pH}=7.4)$. The prepolymer concentration was 10 , 15 , and $20 \%(\mathrm{w} / \mathrm{v})$, respectively. The Gel-ADH and OSA solution were added to a glass bottle to prepare the four types of hydrogels. The volume ratio of the Gel-ADH and OSA solution was $1: 1$ and $2: 1$, respectively. The prepared hydrogels were represented as $10 \%$ Gel-ADH/OSA 1:1, 15\% Gel-ADH/OSA 1:1, 20\% Gel-ADH/OSA 1:1, and 20\% GelADH/OSA 2:1 accordingly.

\section{Gelation Time of the Gel-ADH/OSA Hydrogel}

Gelation time was measured at room temperature by tilt tests. Briefly, four abovementioned types of mixed solutions were injected into glass test tubes immediately after preparation. The tube was tilted horizontally every second, and then, the time when the solution did not have any flow was recorded as the gelation time.

\section{Swelling Behavior of the Gel-ADH/OSA Hydrogel}

The hydrogel samples were lyophilized and immersed in PBS (pH $=7.4$ ). The initial weight of the lyophilized hydrogel was recorded as $W_{0}$. After 12 and $24 \mathrm{~h}$, the hydrogel samples were taken out from the PBS solution and the PBS on the surface of the hydrogels was wiped with filter papers. The weight of the hydrogels at predetermined intervals was recorded as $W_{t}$. The swelling ratio was calculated as follows:

$$
\text { Swelling ratio }(\%)=\frac{W_{t}-W_{0}}{W_{0}} \times 100 \% \text {. }
$$

\section{Micromorphology of the Gel-ADH/OSA Hydrogel}

The microstructure of the lyophilized Gel-ADH/OSA hydrogel was observed by SEM (XL30 ESEM, Philips, Netherlands). The freeze-dried hydrogels were put on a platform and coated with a 
gold layer. Then, the cross-sectional morphology images were observed by SEM.

\section{Hemolytic Activity of the Gel-ADH/OSA Hydrogel}

The erythrocytes were extracted from the fresh mouse blood by centrifugation (1,000 rpm, $5 \mathrm{~min}$ ) and diluted to $5 \%(\mathrm{v} / \mathrm{v})$ with PBS solution $(\mathrm{pH}=7.4)$. The prepared four types of hydrogels were mixed with the erythrocytes solution and incubated for $3 \mathrm{~h}$ $\left(37^{\circ} \mathrm{C}\right)$. Finally, the mixtures were centrifuged at $2,500 \mathrm{rpm}$ for $10 \mathrm{~min}$ and the absorbance of the supernatant from each tube was obtained by using a microplate reader (MK3, Thermo, United States ) at $540 \mathrm{~nm}$. The percentage of hemolysis was calculated as follows:

$$
\text { Hemolysis (\%) }=\frac{O D_{\mathrm{m}}-O D_{p}}{O D_{\mathrm{w}}-O D_{p}} \times 100 \%,
$$

where $O D_{p}, O D_{w}$, and $O D_{m}$ were the absorbance of the supernatant obtained from the erythrocytes mixture with PBS, ultrapure $\mathrm{H}_{2} \mathrm{O}$, and prepared hydrogels, respectively. Cells treated with the PBS solution were used as a positive control, while cells treated with $\mathrm{H}_{2} \mathrm{O}$ were used as a negative control. The hemolytic activity was measured in triplicate.

\section{Cytocompatibility of the Gel-ADH/OSA Hydrogel}

The cytocompatibility of the hydrogels to L929 cells was evaluated by the CCK-8 method (Zhang et al., 2020). Briefly, the cells were incubated in DMEM supplemented with 10\% FBS and $1 \%$ penicillin-streptomycin solution at $37^{\circ} \mathrm{C}$ with $5 \% \mathrm{CO}_{2}$. The four abovementioned types of hydrogels were prepared in 96-well plates at $37^{\circ} \mathrm{C}$, and the cells $\left(5 \times 10^{3}\right.$ cells/well $)$ were cultured with the hydrogels. After 1 and 3 days, $10 \mu$ of CCK- 8 was added to each well and incubated for another $2 \mathrm{~h}$. Finally, the absorbance of each well was obtained by using a microplate reader (MK3, Thermo, United States ) at $450 \mathrm{~nm}$. The hydrogelfree DMEM was used as a control. The viability of cells was measured in triplicate.

\section{Rheological Analysis}

The rheological measurements of the hydrogels were conducted by using a rheometer (MCR 302, Anton Paar, Austria) at $25^{\circ} \mathrm{C}$. A $20 \%$ Gel-ADH/OSA 1:1 hydrogel was placed on a $25 \mathrm{~mm}$ diameter parallel plate of the rheometer. A shear-thinning test using the Gel-ADH/OSA adhesive was measured with a shear rate in the range of $0-2001 / \mathrm{s}$.

\section{Adhesive Capacity of the Gel-ADH/OSA Hydrogel}

A tissue adhesion capacity test was performed using fresh porcine skins. The porcine skins were immersed in PBS $(\mathrm{pH}=7.4$ ) overnight before use. Then, the porcine skins were cut into two oblong samples $(6 \mathrm{~cm} \times 1.5 \mathrm{~cm})$. A $200 \mu \mathrm{l}$ of $\mathrm{Gel}-\mathrm{ADH}$ solution was applied on the first porcine skin, and $200 \mu$ of OSA solution was applied on the second porcine skin. After that, two porcine skins were overlapped and the contacting area was $2 \mathrm{~cm} \times$ $1.5 \mathrm{~cm}$. Finally, the porcine skins were loaded $50 \mathrm{~g}$ for $5 \mathrm{~min}$. The adhesion capacity $(\mathrm{kPa})$ was recorded with a universal tester at a rate of $10 \mathrm{~mm} / \mathrm{min}$.

\section{Statistical Analysis}

All samples were measured in triplicate, and the data were represented as mean \pm standard deviation (SD). All data were analyzed by one-way analysis of variance (ANOVA), and a value of $p<0.05$ was considered to be statistically significant.

\section{RESULTS AND DISCUSSION}

\section{Synthesis and Characterization of OSA and Gel-ADH}

The preparation scheme of OSA and Gel-ADH is shown in Figure 1. Figure 2A shows the FTIR spectra of SA and OSA. Compared with the spectra of SA, a new absorption peak at $1726 \mathrm{~cm}^{-1}$ which is due to the aldehyde group was observed in OSA. The oxidation degree of OSA used in this study was about $79 \%$. The ${ }^{1} \mathrm{H}$ NMR spectra of Gel and Gel-ADH are shown in Figure 2B. Compared with the curve of Gel, new methylene peaks of $\mathrm{ADH}$ at 2.07 and $1.46 \mathrm{ppm}$ were found in the Gel-ADH, indicating that gelatin was successfully modified with adipic acid dihydrazide. The degree of substitution (DS) of $\mathrm{ADH}$, defined as the number of $\mathrm{ADH}$ moieties per 100 carboxyl groups of gelatin, was determined from the ${ }^{1} \mathrm{H}$ NMR spectra (Figure 2B) by comparing the integrals of signals at $1.46\left(\mathrm{~A}_{1.46}\right)$ and $2.60 \mathrm{ppm}$ $\left(\mathrm{A}_{2.60}\right)$. DS, which was expressed as $\mathrm{A}_{1.46} /\left(\mathrm{A}_{1.46}+\mathrm{A}_{2.60}\right) \times 100 \%$ (Yan et al., 2017), was calculated to be $67 \%$.

\section{Characterization of the Gel-ADH/OSA Hydrogel}

Without further treatment, the sol-gel transition of the Gel-ADH/ OSA hydrogel occurred relatively fast, as indicated by tilt tests (Figure 3A). Meanwhile, the hydrogel adhesive developed here was completely transparent and would not cover the underlying tissues when used by surgeons. Figure 3B shows the gelation time of the Gel-ADH/OSA hydrogel with different components and concentrations. The gelation time of $10 \%$ Gel-ADH/OSA 1:1, 10\% Gel-ADH/OSA 1:1, 15\% Gel-ADH/OSA 1:1, and 20\% GelADH/OSA 2:1 was $16.7,11.3,8.7$, and $9.3 \mathrm{~s}$, respectively. For wound sealing, the gelation time of the hydrogel adhesive can affect the hemostasis of damaged tissues. Some commercial tissue adhesives have strong adhesion capacity, but the gelation time is too slow, which may not be enough to stop bleeding in the event of a sudden loss of blood (Qiao et al., 2021). Here, the gelation time of 20\% Gel-ADH/OSA 1:1 and 20\% Gel-ADH/OSA 2:1 was under $10 \mathrm{~s}$. The Gel-ADH/OSA hydrogel with higher concentration had more amino and aldehyde groups to accelerate the Schiff base reaction rate.

An appropriate swelling ratio is an important prerequisite for medical tissue adhesive to maintain a lasting and stable adhesion 


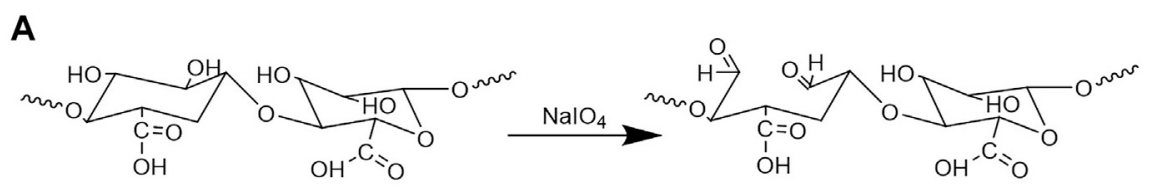

B

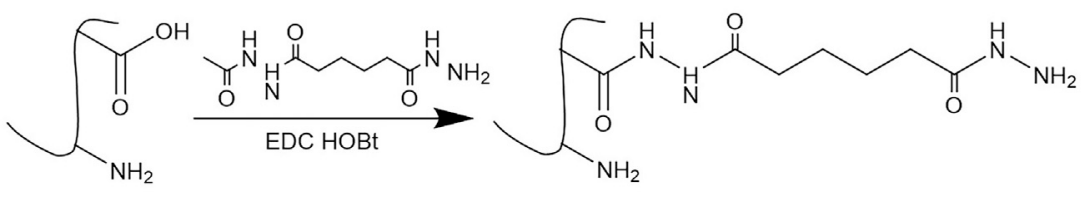

FIGURE 1 | Synthesis of (A) oxidized sodium alginate (OSA) and (B) adipic acid dihydrazide-modified gelatin (Gel-ADH).

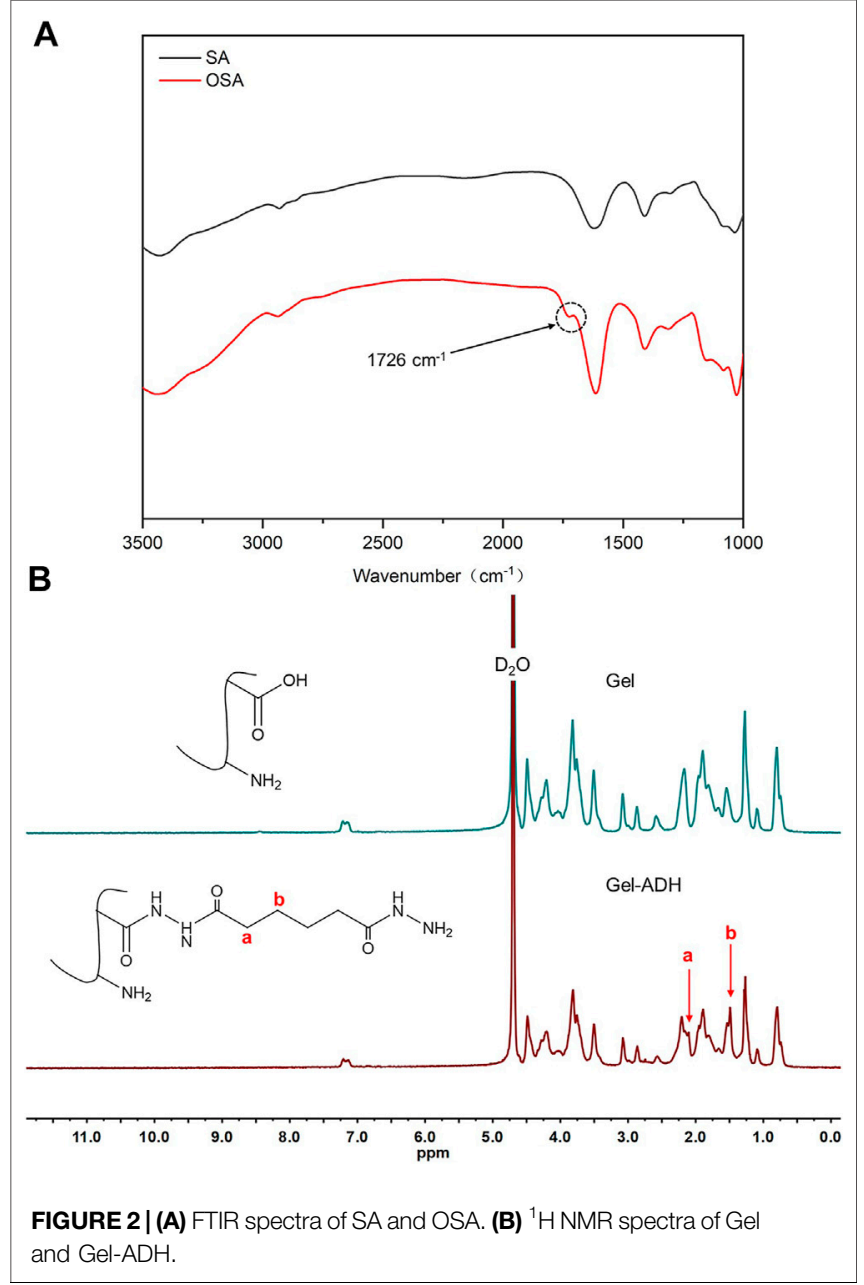

effect in vivo (Liang et al., 2021). If the swelling ratio is too high, the adhesive will squeeze the trauma area, which is not conducive to the adhesion of the wound. The swelling ratio of Gel-ADH/ OSA adhesive is dependent on the prepolymer component and concentration. As shown in Figure 3C, with the increase of prepolymer concentration or the content of Gel-ADH, the swelling ratio decreased which may be caused by the increased internal cross-linking sites in the system. This result indicated that the dense network restricted the expansion of the hydrogel. The swelling ratio of 15\% Gel-ADH/OSA 1:1, 20\% Gel-ADH/ OSA $1: 1$, and $20 \%$ Gel-ADH/OSA $2: 1$ was under $1,000 \%$. However, the swelling ratio of $10 \%$ Gel-ADH/OSA 1:1 decreased after $24 \mathrm{~h}$, probably because of its low concentration, resulting in faster rate of degradation. All these studies proved that Gel-ADH/OSA had good swelling ratio and excellent biodegradability.

Figure 3D shows the internal microscopic morphology of the lyophilized hydrogel characterized by scanning electron microscopy. With the increase of the Gel-ADH component and prepolymer concentration, the porosity decreased gradually. Meanwhile, a uniform microstructure usually provides better mechanical properties theoretically for hydrogels (Pourjavadi et al., 2020). The network structure of Gel-ADH/OSA adhesive presented a high degree of homogeneity.

\section{Biocompatibility of the Gel-ADH/OSA Hydrogel In Vitro}

It is well known that one of the key factors hindering the wide application of biomedical adhesives is their toxic and side effects on cells or their degradation products that can lead to wound tissue infection and necrosis. To investigate the biocompatibility of the Gel-ADH/OSA hydrogel, L929 cells were employed and directly incubated with the Gel-ADH/OSA hydrogel. Then, the CCK-8 assay and live/dead staining were performed on the first and third day of incubation (Figures 4A,B). Compared to that of the control group, cells cultured with Gel-ADH/OSA hydrogels with different compositions and concentrations showed a survival rate higher than $90 \%$ at both day 1 and day 3, indicating the hydrogels have no obvious negative effect on cell viability. This result was also consistent with the live/dead staining (Figure 4B). Most of the cells were stained green in all the groups.

Meanwhile, a hemolysis test was carried out to investigate the hemocompatibility of Gel-ADH/OSA hydrogels. The gross appearance of the erythrocytes after been treated with the solution of PBS, Gel-ADH/OSA hydrogels with 

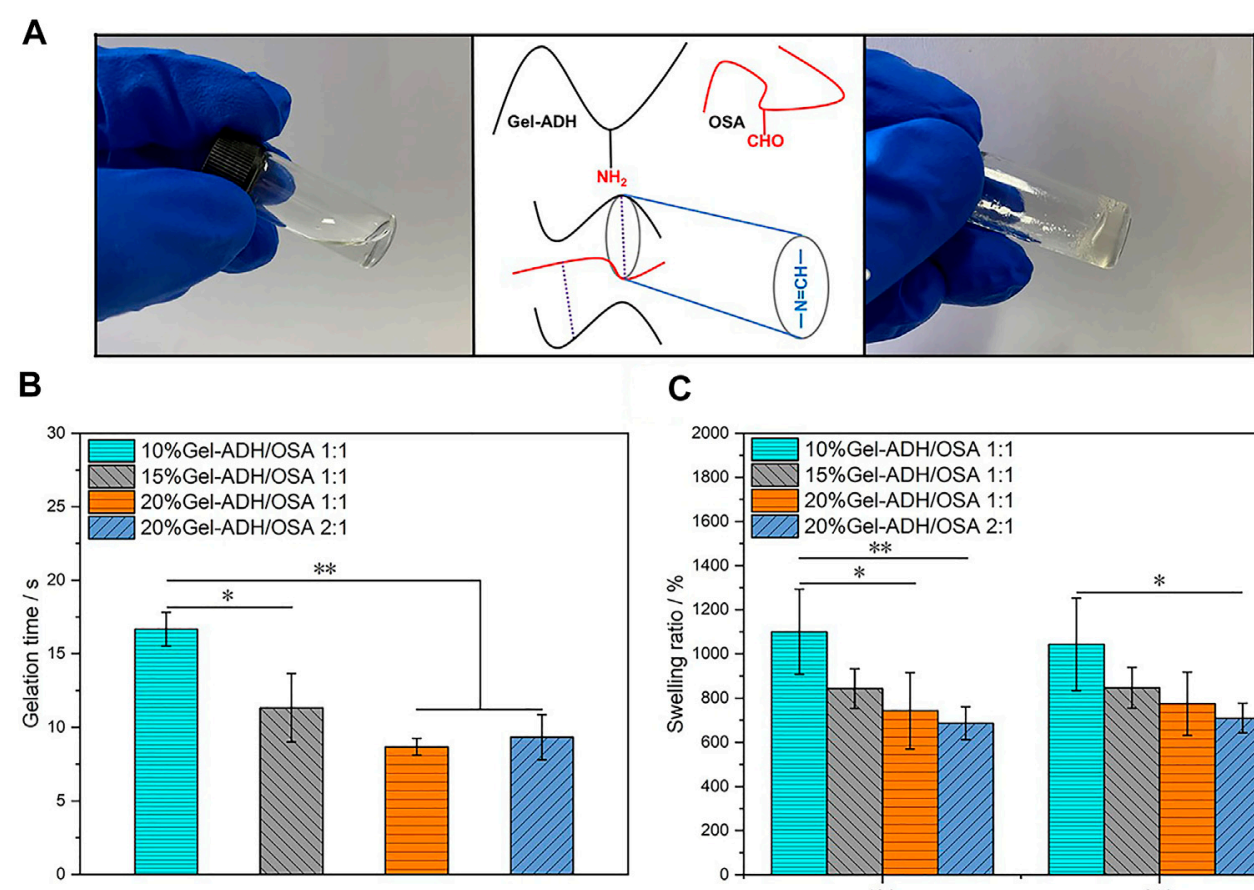

C

\section{D}
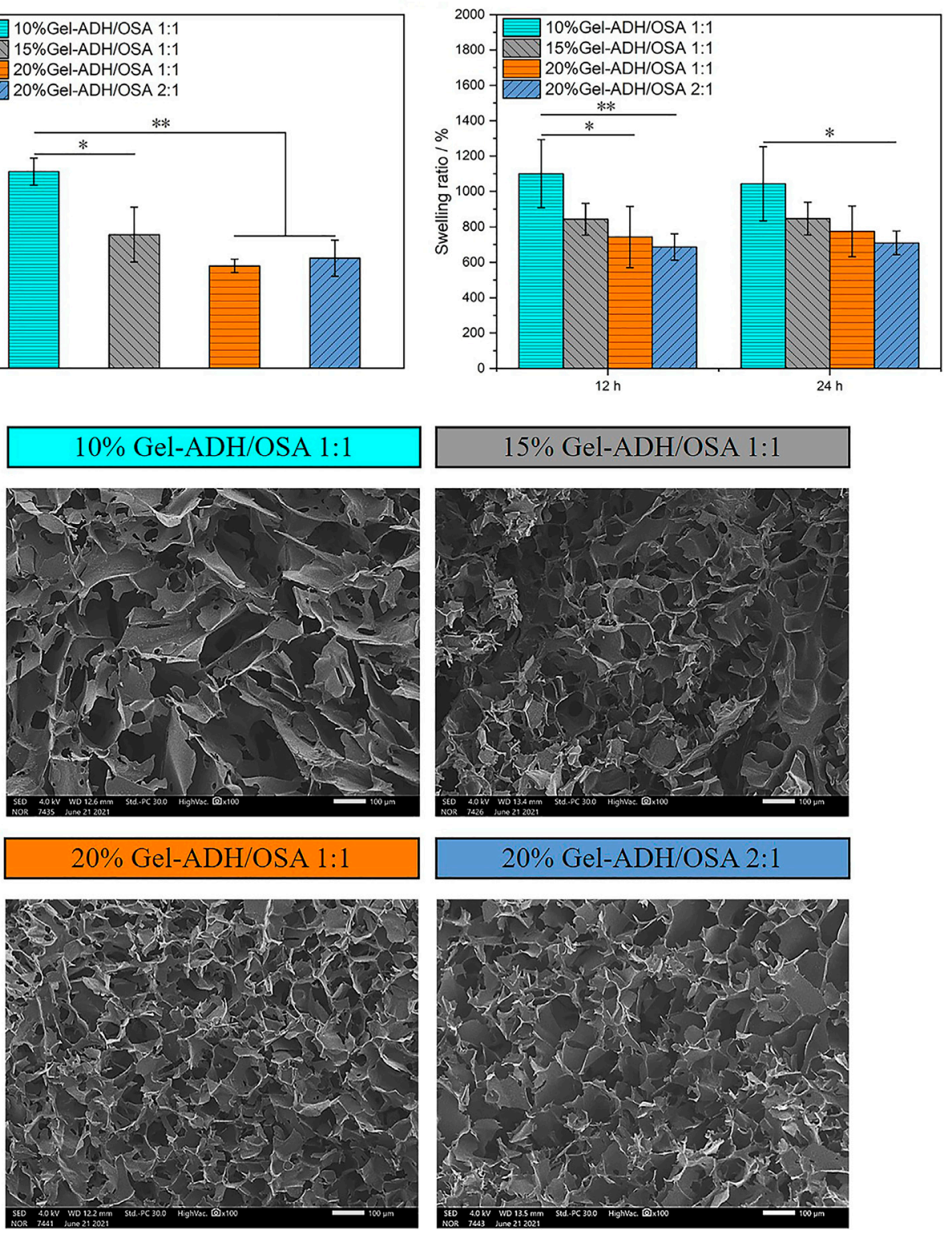

FIGURE 3 | (A) Schematic illustration of the preparation of Gel-ADH/OSA hydrogels. (B) Gelation time, (C) swelling ratio, and (D) SEM images of Gel-ADH/OSA hydrogels with different components and concentrations (scale bar $=100 \mu \mathrm{m},{ }^{*} p<0.05,{ }^{* *} p<0.01, n=3$ ).

different compositions and concentrations, and pure water is shown in Figure 4C. The solutions from Gel-ADH/OSA hydrogels were found to be light red, which was similar to those of the PBS group, while the water group showed bright red color. Figure 4D shows the quantitative analysis result of the hemolysis test, and the hemolysis ratios of Gel-ADH/OSA 
A

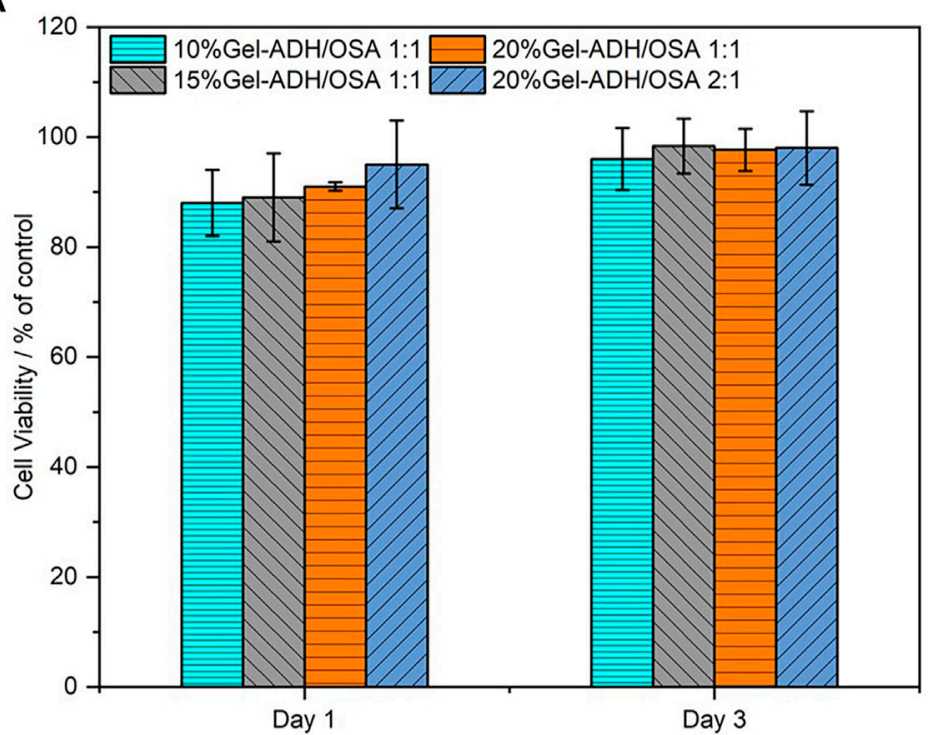

C

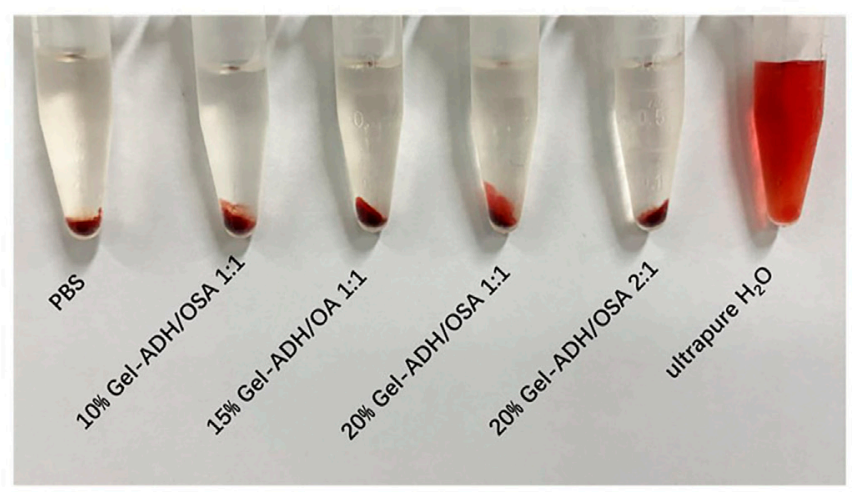

B $20 \%$ Gel-ADH/OSA 1:1
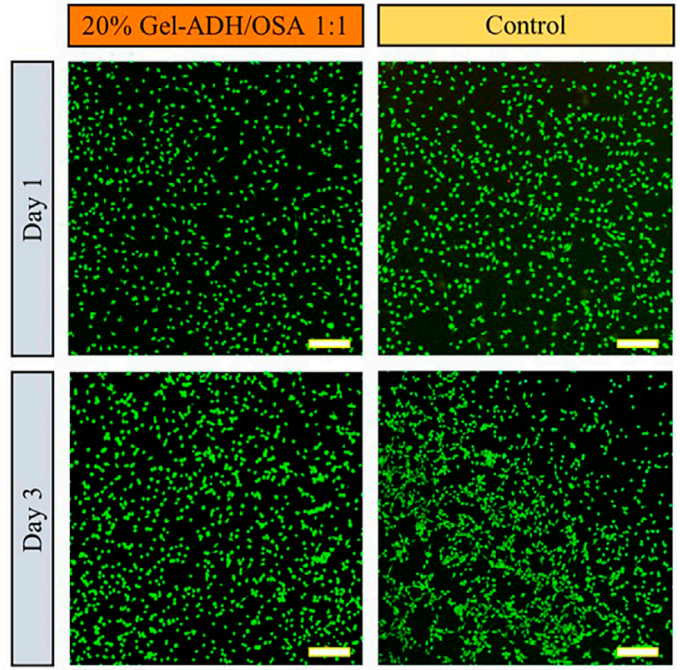

D

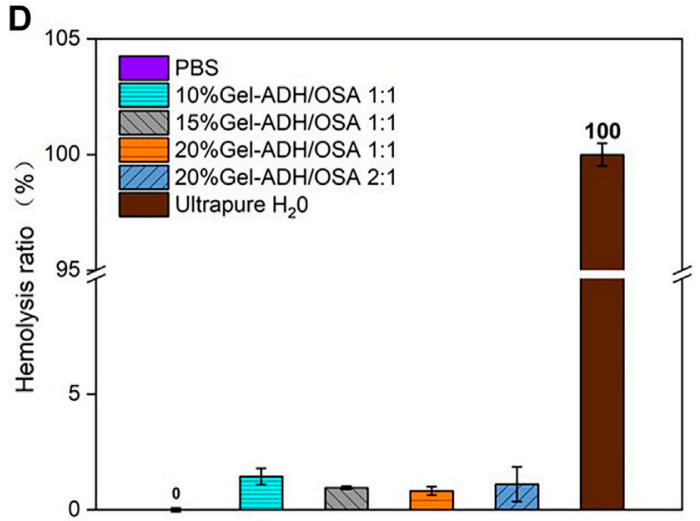

FIGURE 4 | (A) Cell viability assay of L929 cells after being incubated with Gel-ADH/OSA hydrogels with different components and concentrations for 1 and 3 days (B) Live/dead staining of L929 cells after being incubated with the 20\% Gel-ADH/OSA 1:1 hydrogel for 1 and 3 days (scale bar = 100 mm). (C) Image and (D) quantitative analysis of the hemolytic activity of Gel-ADH/OSA hydrogels with different components and concentrations.

hydrogels were $0.82-1.44 \%$, indicating Gel-ADH/OSA hydrogels have excellent hemocompatibility.

\section{Rheological and Tissue Adhesion Capacity of Gel-ADH/OSA Hydrogels}

To check whether the Gel-ADH/OSA adhesive has a shearthinning property, a static shear rate sweep was performed. As shown in Figure 5A, with the increase of shear rate, the viscosity of the hydrogel decreased rapidly. When the shearing rate was $151 / \mathrm{s}$, the $10 \%$ Gel-ADH/OSA 1:1 and $20 \%$ Gel-ADH/OSA 2:1 had a low viscosity of 18 Pas. When the shearing rate was $181 / \mathrm{s}$, the $15 \%$ Gel-ADH/OSA 1:1 and $20 \%$ Gel-ADH/OSA 1:1 had a low viscosity of 9 Pas. Therefore, the Gel-ADH/OSA adhesive was shear-thinning because of dynamic Schiff base covalent bonding. As shown in Figure 5B, the Gel-ADH/OSA adhesive had excellent injectability in the process of application (Yang et al., 2020).
To explore the adhesion capacity of Gel-ADH/OSA hydrogels in vitro, standard shear and peer tests were performed (Suneetha et al., 2019) (Figures 5C,D). As shown in Figure 5, with the increase of prepolymer concentration, the shear and peer stress of Gel-ADH/OSA hydrogels increased significantly. That is because the gelatin and sodium alginate are sticky inherently, and the Gel$\mathrm{ADH} / \mathrm{OSA}$ hydrogel with higher concentration had more amino and aldehyde groups to accelerate the Schiff base reaction rate. Meanwhile, the high oxidation degree of OSA would accelerate cross-linked reactions and improve the adhesive strength of the hydrogel. Since the 20\% Gel-ADH/OSA 1:1 has more sites to react with amino groups on the surface of skin tissue, the lap shear strength $(45 \pm 14 \mathrm{kPa})$ and peel stress $(28 \pm 6 \mathrm{kPa})$ were higher than those of other groups, including the commercial adhesive Porcine Fibrin Sealant Kit. Previous studies also showed that the Fibrin Sealant has limited adhesion strength (Chow et al., 2021). To further confirm the biomedical application potential of the hydrogel adhesive, wound sealing and healing investigation will be performed in the future animal studies. 

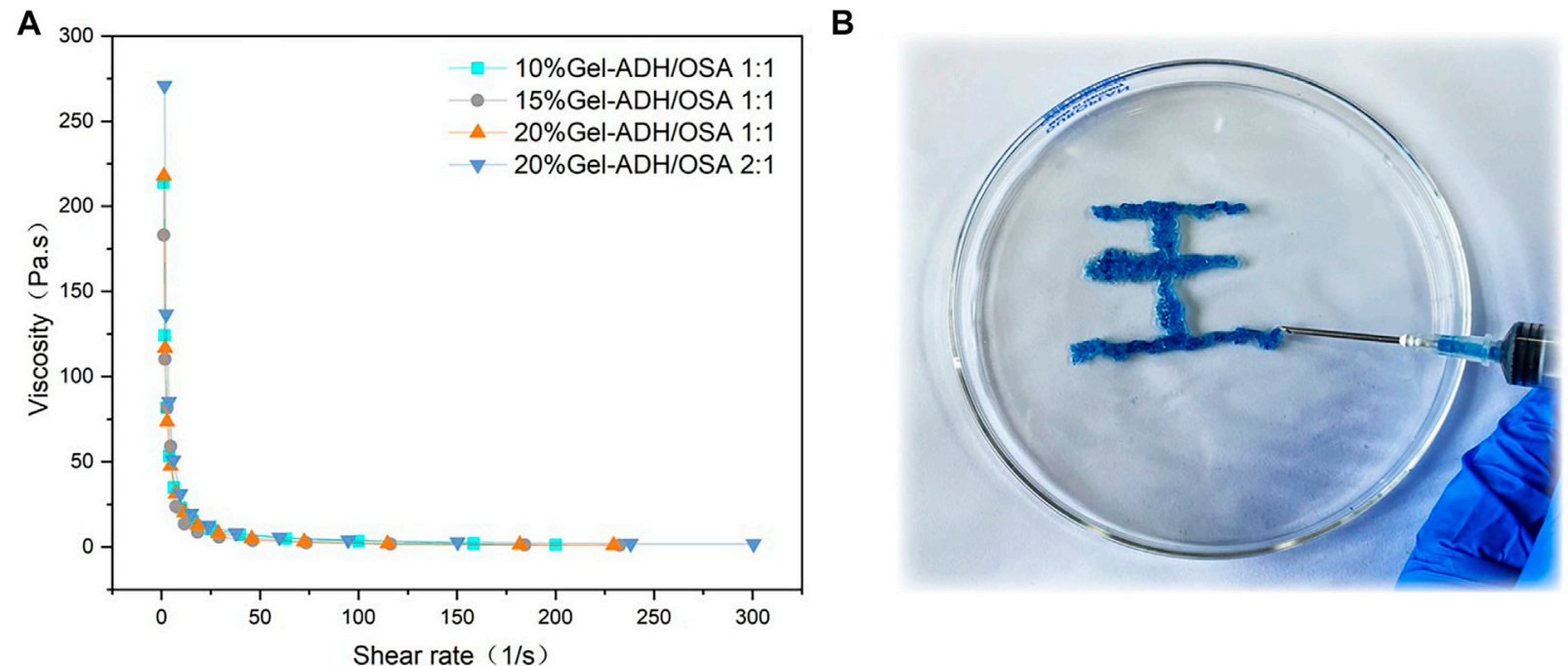

C
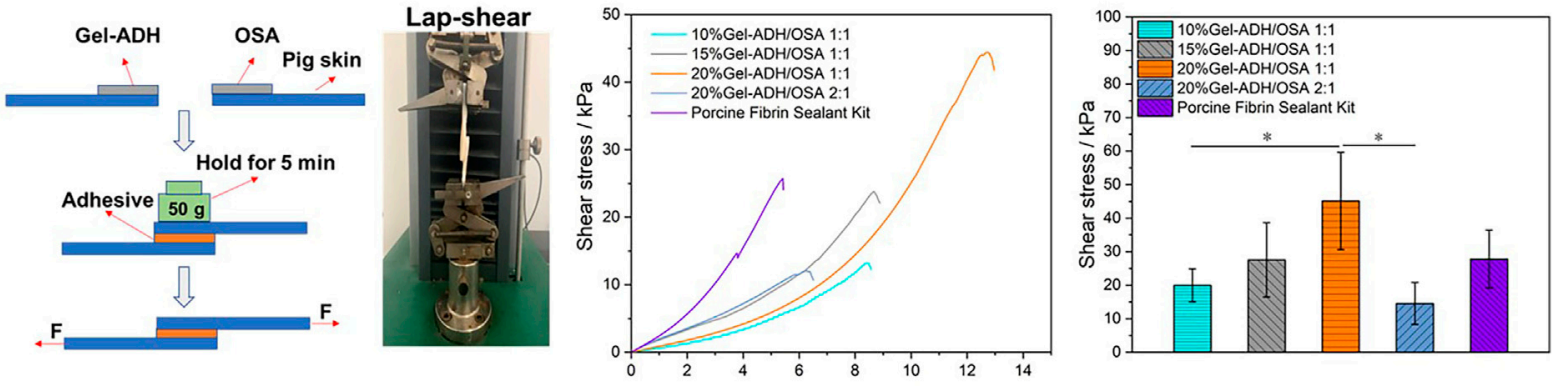

D
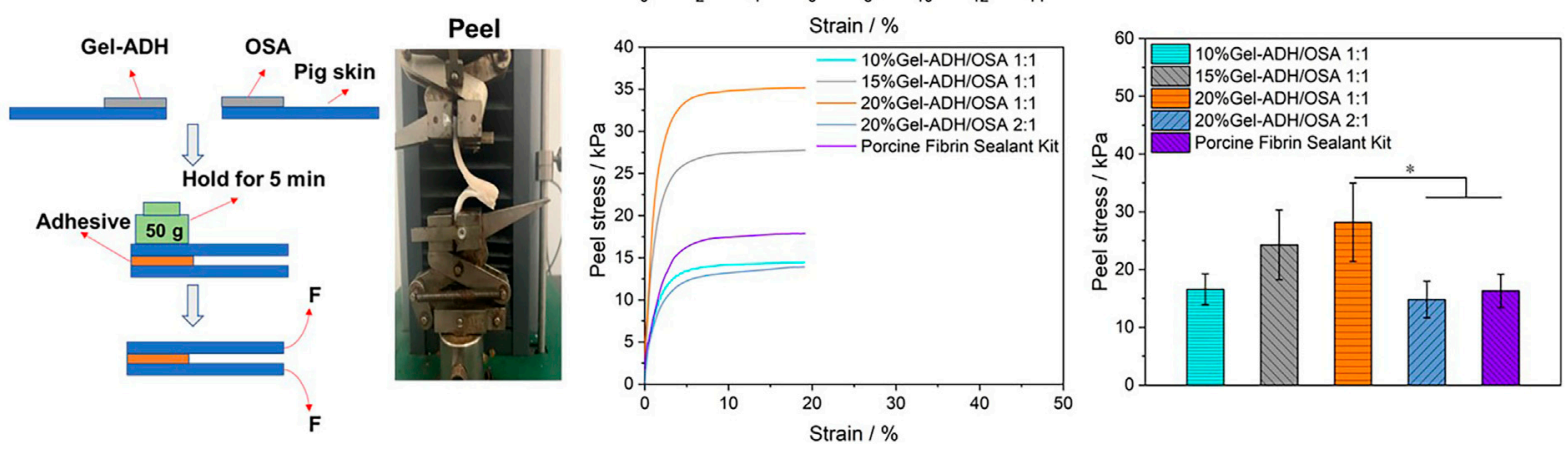

FIGURE 5 | (A) Shear-thinning ability of Gel-ADH/OSA hydrogels with different components and concentrations. (B) Injectability of the 20\% Gel-ADH/OSA 1:1 hydrogel. A schematic and quantitative analysis of (C) a standard lap shear test and (D) a standard peel test using Gel-ADH/OSA hydrogels with different components and concentrations. A commercialized Porcine Fibrin Sealant Kit served as the control ( $\left.{ }^{*} p<0.05, n=3\right)$.

\section{CONCLUSION}

In this study, an injectable hydrogel tissue adhesive was developed, and the hydrogel showed a suitable swelling ratio, good injectability, and excellent biocompatibility. We also found that the adhesion capacity was related to the prepolymer component and concentration. The $20 \%$ GelADH/OSA 1:1 hydrogel showed the highest adhesion ability, which was also higher than that of the commercial Porcine Fibrin Sealant Kit. All these results suggested that the polysaccharide-based hydrogel was a promising candidate for a soft tissue adhesive.

\section{DATA AVAILABILITY STATEMENT}

The original contributions presented in the study are included in the article/Supplementary Material; further inquiries can be directed to the corresponding authors.

\section{AUTHOR CONTRIBUTIONS}

$\mathrm{HM}$ and ZG contributed to the conception and design of the study. YX, HX, and SH performed the experiments. YX wrote the first draft of the article. $\mathrm{HX}, \mathrm{SH}$, and $\mathrm{HZ}$ wrote sections of 
the article. HM, XQ, YH, and ZG performed the statistical analysis and writing-review and editing. All authors contributed to article revision, read, and approved the submitted version.

\section{FUNDING}

This work was supported by the Key R\&D Plan of Jiangsu Province (BE2018010-3), the National Natural Science

\section{REFERENCES}

Chow, N., Miears, H., Cox, C., and MacKay, B. (2021). Fibrin Glue and its Alternatives in Peripheral Nerve Repair. Ann. Plast. Surg. 86 (1), 103-108. doi:10.1097/sap.0000000000002408

Du, X., Hou, Y., Wu, L., Li, S., Yu, A., Kong, D., et al. (2020). An Anti-infective Hydrogel Adhesive with Non-swelling and Robust Mechanical Properties for Sutureless Wound Closure. J. Mater. Chem. B 8 (26), 5682-5693. doi:10.1039/ d0tb00640h

Gao, L., Chen, J., Feng, W., Song, Q., Huo, J., Yu, L., et al. (2020). A Multifunctional Shape-Adaptive and Biodegradable Hydrogel with Hemorrhage Control and Broad-Spectrum Antimicrobial Activity for Wound Healing. Biomater. Sci. 8 (24), 6930-6945. doi:10.1039/d0bm00800a

Geng, H., Cui, J., and Hao, J. (2020). Mussel-Inspired Hydrogels for Tissue Healing. Acta Chim. Sinica 78 (2), 105-113. doi:10.6023/a19080301

Gillman, N., Lloyd, D., Bindra, R., Ruan, R., and Zheng, M. (2020). Surgical Applications of Intracorporal Tissue Adhesive Agents: Current Evidence and Future Development. Expert Rev. Med. Devices 17 (5), 443-460. doi:10.1080/ 17434440.2020 .1743682

Gowda, A. H. J., Bu, Y., Kudina, O., Krishna, K. V., Bohara, R. A., Eglin, D., et al. (2020). Design of Tunable Gelatin-Dopamine Based Bioadhesives. Int. J. Biol. Macromolecules 164, 1384-1391. doi:10.1016/j.ijbiomac.2020.07.195

Hozumi, T., Kageyama, T., Ohta, S., Fukuda, J., and Ito, T. (2018). Injectable Hydrogel with Slow Degradability Composed of Gelatin and Hyaluronic Acid Cross-Linked by Schiffs Base Formation. Biomacromolecules 19 (2), 288-297. doi:10.1021/acs.biomac.7b01133

Ke, X., Dong, Z., Tang, S., Chu, W., Zheng, X., Zhen, L., et al. (2020). A Natural Polymer Based Bioadhesive with Self-Healing Behavior and Improved Antibacterial Properties. Biomater. Sci. 8 (15), 4346-4357. doi:10.1039/ d0bm00624f

Li, D. W., Chen, J., Wang, X., Zhang, M. M., Li, C. L., and Zhou, J. (2020). Recent Advances on Synthetic and Polysaccharide Adhesives for Biological Hemostatic Applications. Front. Bioeng. Biotechnol. 8, 16. doi:10.3389/fbioe.2020.00926

Liang, Y., Li, Z., Huang, Y., Yu, R., and Guo, B. (2021). Dual-Dynamic-Bond CrossLinked Antibacterial Adhesive Hydrogel Sealants with On-Demand Removability for Post-Wound-Closure and Infected Wound Healing. Acs Nano 15 (4), 7078-7093. doi:10.1021/acsnano.1c00204

Lv, C., Li, L., Jiao, Z., Yan, H., Wang, Z., Wu, Z., et al. (2021). Improved Hemostatic Effects by $\mathrm{Fe} 3+$ Modified Biomimetic PLLA Cotton-like Mat via Sodium Alginate Grafted with Dopamine. Bioactive Mater. 6 (8), 2346-2359. doi:10.1016/j.bioactmat.2021.01.002

Neuffer, M. C., McDivitt, J., Rose, D., King, K., Cloonan, C. C., and Vayer, J. S. (2004). Hemostatic Dressings for the First Responder: A Review. Mil. Med. 169 (9), 716-720. doi:10.7205/milmed.169.9.716

Pourjavadi, A., Tavakolizadeh, M., Hosseini, S. H., Rabiee, N., and Bagherzadeh, M. (2020). Highly Stretchable, Self-adhesive, and Self-healable Double Network Hydrogel Based on Alginate/polyacrylamide with Tunable Mechanical Properties. J. Polym. Sci. 58 (15), 2062-2073. doi:10.1002/pol.20200295

Qiao, Z., Lv, X., He, S., Bai, S., Liu, X., Hou, L., et al. (2021). A Mussel-Inspired Supramolecular Hydrogel with Robust Tissue Anchor for Rapid Hemostasis of Arterial and Visceral Bleedings. Bioactive Mater. 6 (9), 2829-2840. doi:10.1016/ j.bioactmat.2021.01.039
Foundation of China (31800803, 31771067, and 32071364), and the Scientific Research Foundation for Talent Introduction of Nanjing Tech University (39803129 and 39803130).

\section{ACKNOWLEDGMENTS}

The authors gratefully acknowledge the Priority Academic Program Development of Jiangsu Higher Education Institutions (PAPD).

Reakasame, S., and Boccaccini, A. R. (2018). Oxidized Alginate-Based Hydrogels for Tissue Engineering Applications: A Review. Biomacromolecules 19 (1), 3-21. doi:10.1021/acs.biomac.7b01331

Suneetha, M., Rao, K. M., and Han, S. S. (2019). Mussel-Inspired Cell/TissueAdhesive, Hemostatic Hydrogels for Tissue Engineering Applications. ACS Omega 4 (7), 12647-12656. doi:10.1021/acsomega.9b01302

Yan, S., Wang, T., Li, X., Jian, Y., Zhang, K., Li, G., et al. (2017). Fabrication of Injectable Hydrogels Based on poly(L-Glutamic Acid) and Chitosan. RSC Adv. 7 (28), 17005-17019. doi:10.1039/c7ra01864a

Yang, B., Song, J., Jiang, Y., Li, M., Wei, J., Qin, J., et al. (2020). Injectable Adhesive Self-Healing Multicross-Linked Double-Network Hydrogel Facilitates FullThickness Skin Wound Healing. ACS Appl. Mater. Inter. 12 (52), 57782-57797. doi:10.1021/acsami.0c18948

Yang, X., Liu, W., Li, N., Wang, M., Liang, B., Ullah, I., et al. (2017). Design and Development of Polysaccharide Hemostatic Materials and Their Hemostatic Mechanism. Biomater. Sci. 5 (12), 2357-2368. doi:10.1039/c7bm00554g

Yuan, L., Fan, W., Han, L., Guo, C., Yan, Z., Zhu, M., et al. (2018). Evaluation of Hydrogels for Soft Tissue Adhesives In Vitro and In Vivo Analyses. Front. Mater. Sci. 12 (1), 95-104. doi:10.1007/s11706-018-0405-4

Zhang, C., Yang, X., Hu, W., Han, X., Fan, L., and Tao, S. (2020). Preparation and Characterization of Carboxymethyl Chitosan/collagen Peptide/oxidized Konjac Composite Hydrogel. Int. J. Biol. Macromolecules 149, 31-40. doi:10.1016/ j.ijbiomac.2020.01.127

Zhao, X., Hu, D. A., Wu, D., He, F., Wang, H., Huang, L. J., et al. (2021). Applications of Biocompatible Scaffold Materials in Stem Cell-Based Cartilage Tissue Engineering. Front. Bioeng. Biotechnol. 9, 23. doi:10.3389/ fbioe.2021.603444

Zhou, J., Wu, Y., Zhang, X., Lai, J., Li, Y., Xing, J., et al. (2021). Enzyme Catalyzed Hydrogel as Versatile Bioadhesive for Tissue Wound Hemostasis, Bonding, and Continuous Repair. Biomacromolecules 22 (4), 1346-1356. doi:10.1021/ acs.biomac.0c01329

Zhu, H., Mei, X., He, Y., Mao, H., Tang, W., Liu, R., et al. (2020). Fast and High Strength Soft Tissue Bioadhesives Based on a Peptide Dendrimer with Antimicrobial Properties and Hemostatic Ability. ACS Appl. Mater. Inter. 12 (4), 4241-4253. doi:10.1021/acsami.9b18720

Conflict of Interest: The authors declare that the research was conducted in the absence of any commercial or financial relationships that could be construed as a potential conflict of interest.

Publisher's Note: All claims expressed in this article are solely those of the authors and do not necessarily represent those of their affiliated organizations, or those of the publisher, the editors, and the reviewers. Any product that may be evaluated in this article, or claim that may be made by its manufacturer, is not guaranteed or endorsed by the publisher.

Copyright (c) 2021 Xing, Qing, Xia, Hao, Zhu, He, Mao and Gu. This is an openaccess article distributed under the terms of the Creative Commons Attribution License (CC BY). The use, distribution or reproduction in other forums is permitted, provided the original author(s) and the copyright owner(s) are credited and that the original publication in this journal is cited, in accordance with accepted academic practice. No use, distribution or reproduction is permitted which does not comply with these terms. 